\title{
FIBROELASTOSIS DEL ENDOCARDIO
}

\section{DrS. FERNANDO GONZALEZ, ALFONSO TOBAR y EUGENIO VALENZUELA Unidad Cardiovascular del Hospital "Entique Deformes". Valparaiso.}

Entre las causas de insuficiencia cardíaca del lactante, hay un cuadro que ha venido adquiriendo cada vez más importancia en el último decenio y es la llamada por Weinberg y Himelfarb ${ }^{1}$, fibroelastosis del endocardio, denominación que parece la más acertada y que ha sido adoptada por la mayor parte de los autores que se han preocupado de esta afección. También se denomina, simplemente, esclerosis o fibrosis del endocardio. Es una enfermedad que se ha separado de las llamadas cardiomegalias Congénitas o idiopáticas del lactante y que actualmente es una entidad patológica autónoma, si bien aún su etiología y descripción clínica permanecen confusas.

La suerte de haber podido observar dos casos, los cuales creemos sean los primeros relatados en nuestro país, son el motivo de esta presentación.

Caso 1. A. P. V. Obs. No 54/8736.

Niña de 5 mesos, que es hospicalizada en la Posta Infuntil en Enero de 1954, por presentar desde hace 24 horas palidez, inquietud, disnea. vomitos $e$ ina. petencia. Desde 20 dias antes la notaban decaida, anoréxica $y$ con llanto fracuente.

En sus antecedentes. amenaza de aborto at segundo mes del embarazo. Un hermano falleció a los 11 meses en forma más o menos brasca. de cuadro catalogado como bronconeumonia. Abuelo paterno cardiópata.

En su examen de ingreso se aprecia niña en buenas condiciones nutritivas, con disnca intensa, palidez, acentuada. cianosis de labios y uñas. Temperatura $38^{\circ}$ (rectal). Taquicardia de 150. Al examen cardiaco, tonos taquicárdicos. algo apagados; til to de galope; no se aprecian frémitos ni soplos. Ruidos húmedos en ambas bases pulmonares. Heparomegalia acentuada $(3,5$ cnis. bajo el reborde costal). Femorales laten normales.
Ante el cuadro de insuficiencia cardiaca en un lactante, sé plantea, entre otras. la hipótesis diagnostica de fibroelascosis del endocardio. 5e indica oxigeno, penicilina. digital $y$ cortisona $(12.5 \mathrm{mlgrs}$. cada 6 horas, por vis intramuscular).

Se practica un hemograma que sólo demuestra una desriación a la izquierda de 13 baciliformes.

E'l estudio radiológico nos informa: agrandamiento bilateral de la sombra cardiaca. con la parta redondeada y levantada (teleradiografía en decúbito dorsal). En O.I.A., prominencia del arco ventricular izquierdo. sin agrandamiento de las cavidades derechas. Trama vascular pulmonar acentuada en ambos campos, más visibles a derecha. Pediculo vascular de aspecto normal. Indice cardio-toráxico: 0.68 (Normal: $0.5 \mathrm{I}-0.62$ ). (Figs. 1 \% 2 l.

Fn al E.C.G. observamos taquicardia sinusal. ondas $S$ profundas en las precordiales $y$ ondas $T$ negativas en aVf. V5 y V6: aplarada ed aV1. (Fig. 3).

El niño continúa agravándose, aumentan la cianosis y la disnea. aparece edema en las extremidades inferlores, se llenan ambos campos pulmonares de estcrtores húmedos, se presenta obnubilación sensorial y falkee a las 17 horas de su ingreso.

Autapsia. - Abierto el tórax. pericardio de as. pecto normal, que contiene unos 10 ec. de liquido. Hidrotórax bilateral. Pulmones con hipostasia de lóbulos inferiores. Timo bien desarrollado, pesa $60 \mathrm{grs}$. En la cavidad peritoneal se encuentra $200 \mathrm{cc}$. de li. quido. Cerebto congestionado $y$ edematoso.

Corazón aumentado de tamaño, con un peso de 120 grs. (N:31 grs.). El ventriculo izquierdo aparece dilatado, con músculos panílares aplanados. siendo su grosor a nivel de la punta, de $0.4 \mathrm{~cm}$. El endocardio se aprecia de color grisáceo-blanquecino, engrosado. Auricula izquierda amplia, con endocardio tambien do aspecto blanquecino. Cavidades derechas de aspecto normal. Válvalas normales. Aorta y pulmonar normales. Arterias coronarias con orificios permeables y nacimien. to normales. Miocardio de aspecto normal. (Fig. 4). 


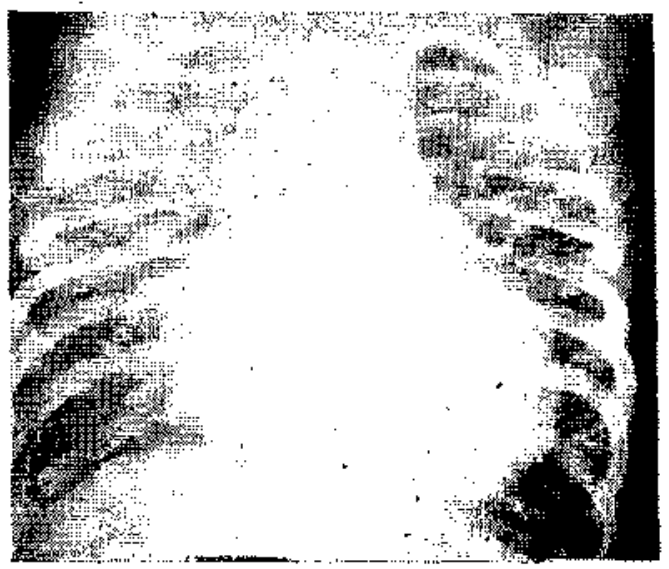

Fig. 1. - Observese el agcandamiento marcado de la sombta cardiaca y la acentuación de la trama rasculat pulmonar.

Al examen mictoscópico del corazón se obscris un acentuado engrosamienlo del endocardio. engrosamicnto constiquido sobre todo por fitras coligenas y clasticas. No hay infiltado inflamatorio. En algunos sitios el cejido fibroelístico endocádico invade las fibras muscularis del miocardio. (Fig. 5).

Caso 2. - C. C. P. Obs. N! 52/8812.

Niño de 2 años 5 meses. que se hospilatiza an Abril de 195? en la Posta Infantil. por presentar clesde bace algunas horas: rómitos y diarress intens.s. inquiztud $y$ disnea. Como antecedentes. solamente relardo de su desarrollo ponderal y an sus funciones eslático-dinamitas. Al examen de ingreso se aprecia niño gravesente enlermo. en malas condiciones nutritivas. deshidratado. disneico. Al examen cardíaco sólo se

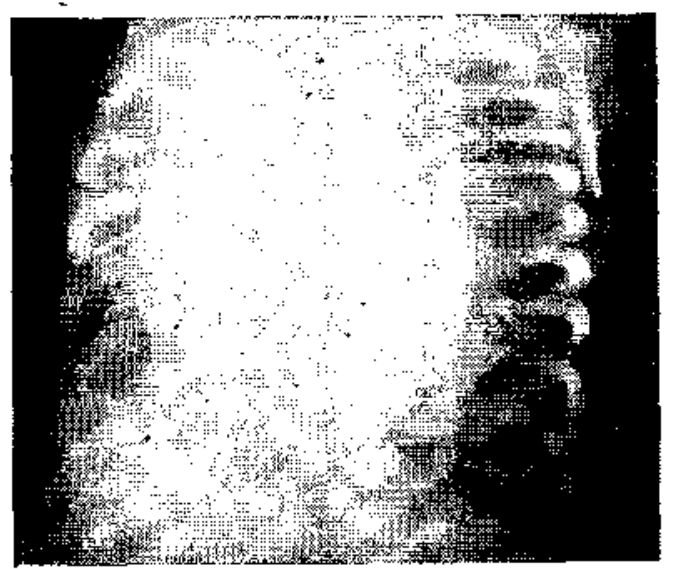

Fig. 2. - Lin posición oblicua izquierda anterior se aprecia la prominencia del arco ventricular izquierdo. que ocupa el espacio retrocardiaco sobrepasundo la columna vertebral. Nótess la falta de agrandamienco de las savidades derechas hacia adelante. pptecia raquicardia. Abdomen depresible, con sensibilidad difusa. Se hacen los diagnósticos de: Intoxicación alimenticia. Enterocolitis. Sindrome tóxico. Se indica hidratación, exirato suptarenal y sulfadiazina. If ni“ fo rallese 8 hotas mas tarde.

Autepsta, - Congestion meningo-cerbrit. Hliperp'asta ganglionar inlertráqueobrónquica y cervical superior e uplerior. Tímo aunensado de lamaño, pusa 20 grs. Pulmones: salida de liquido a su expresión. especirlmemte de los lóbulos inferiors. Corazon: Ad. heren:ias fibrosas marciales del pericardio en la cara antrior jel miocatdio. Aumento global det coraron. favidades cardiacas dilandas. Velos valulares $y$ indotefíc de los grandes vasos nada de especial. Coronarias normales. Llama la atención et endocatdio de las ca. vidadus izquierdas, engrosado y de aspecto blanquecino. fibroso. an forma difusa. El miocardio del ventriculo izquicrdo presenta esclerosis en la parti media de su espeser.
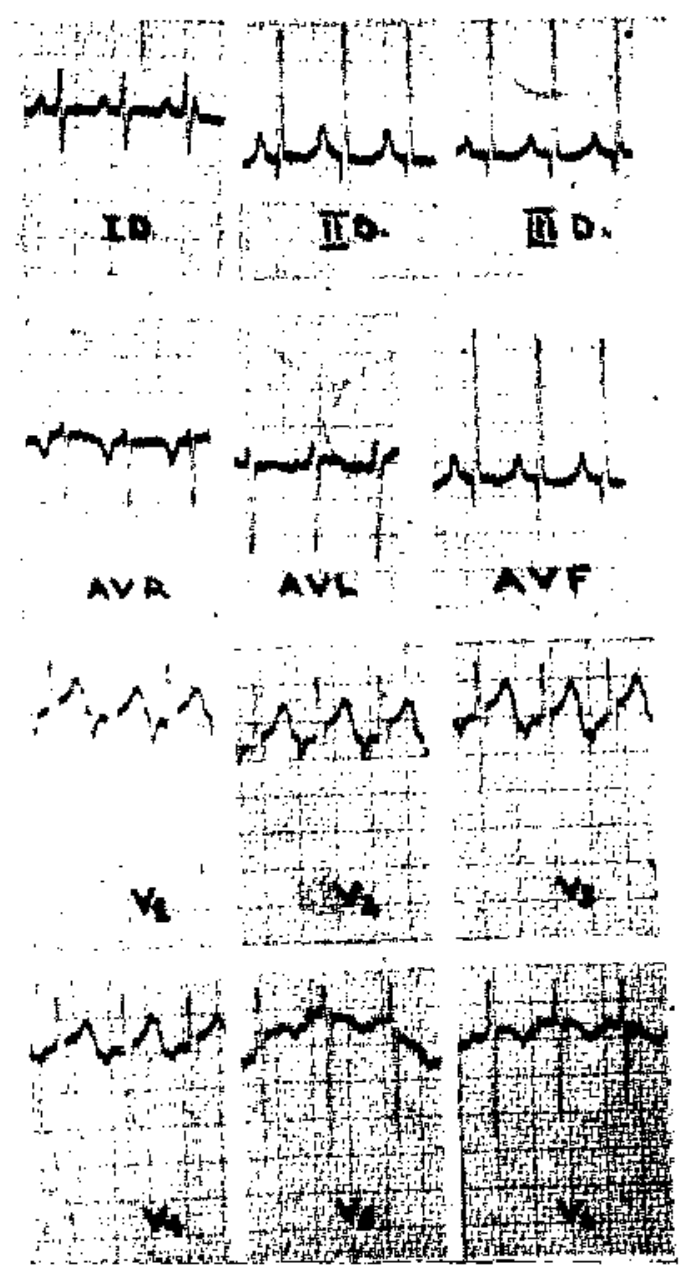

I'ig. 3. - ECG, con $S$ profunda en $V_{1} y$ ondas $T$ negativas en $V_{5} y V_{0}$. 


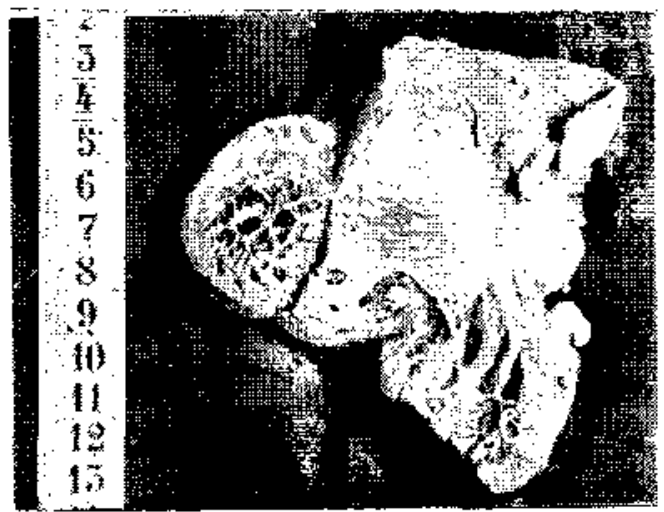

Fig. 4. - Pizza anatómica mostrando el ventriculo jzquierdo abierro. en el cual se aprecia el mareado engrosamicrto del endocardio y ba coloración blancogrisacea nacarada de ciste.

A continuación, basados en el estudio de ouestras observaciones y muy especialmente en el de 77 casos publicados por autores franceses, belgas y sobre todo norteamericanos, cuya bibliografía es, con mucho, la más numerosa, haremos una relación de las lesiones anatómicas y aspecto clínico de esta afección.

\section{Anatomía patológica}

La necropsia muestra generalmente un corazón enorme, cuyo peso alcanza 3 a 5 veces el normal y esto en especial por aumento de volumen del ventriculo izquierdo. Las otras cavidades generalmente están dilatadas. En la mayor parte de los casos la lesión endocárdica afecta exclusivamente las cavidades izquierdas y muy particularmente el ventriculo izquierdo, que en ocasiones es el único alterado.

El endocardio está engrosado en forma muy acentuada, con un aspecto blanco-grisáceo o francamente blanco-aporcelanado, muy característico. Este engrosamiento afecta bastante a menudo las valvas de la mitral y también las sigmoideas aórticas, produciendo en ocasiones diversos grados de estrechez valvular.

Histológicamente, este espesamiento endocárdico se muestra constituído por proliferación importante de fibras colágenas $y$ una gran riqueza de fibras elásticas, especialmente de las capas profundas (Fibroelastosis del Endocardio).

Esta fibrosis del endocardio invade frecuentemente el miocardio subyacente, donde forma fascículos que disocian las

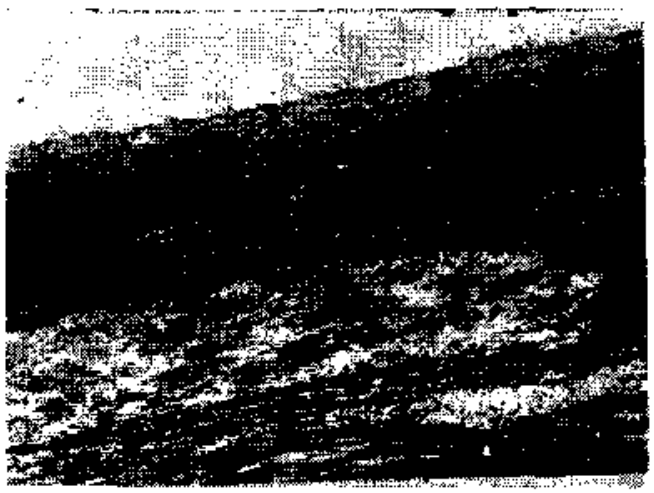

Fig. 5. - Corte microstopico del ventriculo izquierdo. ent a' que sc observa el engrosamiento del endocardio y la inlensa proliferación de fibras elásticas. las cuales en parle invaden el miocardio.

fibras musculares. Llama, si, la atención, una franca desproporción entre la importancia de las lesiones del endocardio y el compromiso relativamente discreto del miocardio. Otro elemento característico de la fibroelastosis, es la ausencia de infiltrados inflamatorios en los tejidos afectados.

En un caso relatado por Stadler y col. " y en uno de los nuestros, existia una fibrosis parcelar del pericardio. Tabla 1$)$.

\section{Cuadro clínico}

La fibroelastosis del endocardio es una enfermedad que afecta principalmente al lactante $y$ al niño menor; la mayor parte de Ios casos se ven antes del año de edad. El factor dominante en esta afección parece ser la aparición de un cuadro de insuficiencia cardíaca aguda, de evolución fatal. Clínicamente, la sintomatología previa a la muerte puede ser de corta evolución, o aún no haber síntomas, produciéndose así aparentemente una muerte súbita, o también, los síntomas pueden evolucionar por meses y aún años, con remisiones transitorias y aparente mejoria, hasta que una acentuación de éstos llevan al nin̄o a la muerte. (Tabla 2 ).

TARTA NY 1

LESIONES ANATOMICAS

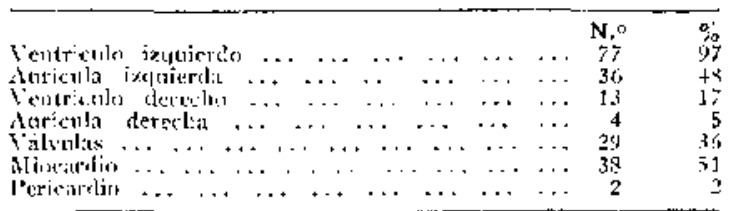


TABLA No 2

\section{EDAD DE LOS PACIENTES Y DURACION} DE LOS SINTOMAS

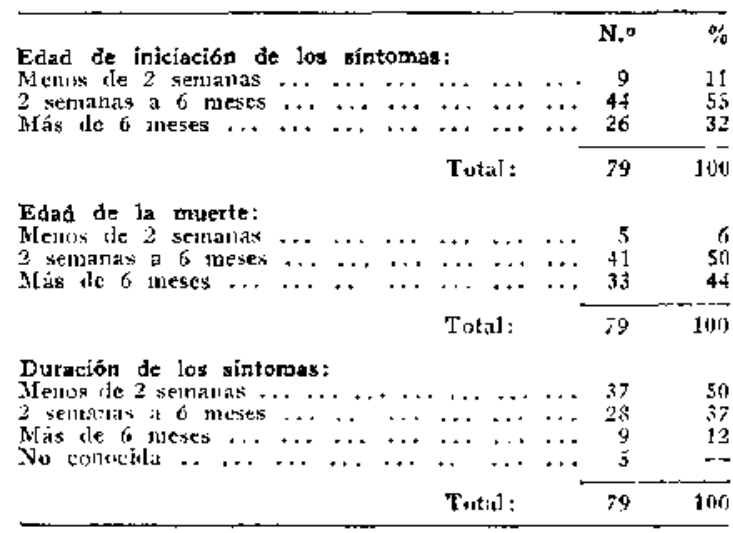

En general, la enfermedad se revela en forma violenta, por un sindrome de desfallecimiento cardíaco hiperagudo, que sobreviene en un lactante hasta entonces aparentemente sano. A continuación de una enfermedad intercurrente $o$ de un esfuerzo cualquiera, el niño se pone intensamente pálido y disneico, hay inquietud, rehusa toda alimentación y a menudo presenta vómitos $\mathrm{y}$ una tos seca, persistente. La cianosis es a menudo discreta, acentuándose en los períodos finales; el pulso es débil y taquicárdico; generalmente la temperatura es normal, o ligeramente elevada. La auscultación da en la mayoría de los casos pocos signos, algunos estertores finos diseminados en ambos campos pulmonares, principalmente en las bases y al examen cardíaco una taquiarritmia con tonos algo apagados; en ocasiones pueden auscultarse soplos sistólicos, generalmente poco intensos. El hígado está aumentado de volumen y puede haber edema periférico. (Tabla 3 ).

EI electrocardiograma en las derivaciones standards muestra sólo escasas alteraciones; se puede observar una desviación del eje eléctrico hacia la izquierda o hacia la derecha según los casos, no exis. tiendo generalmente alargamiento del tiempo de conducción. Pero las derivaciones precordiales muestran en la mayor parte de los casos signos marcados de sobrecarga ventricular izquierda y en particular inversión de $\mathbf{T}$ en V5 y V6 con grandes ondas $\mathrm{R}$ en estas derivaciones $\mathrm{y}$ grandes ondas $\mathbf{S}$ en las precordiales derechas. Se han descrito casos que presentaban bloqueo incompleto de rama izquierda. (Vulliamy ${ }^{3}$, Lewis ${ }^{4}$, Stadler ${ }^{2}$ ).
TABLA Xe 3

MANIFESTACIONES CLINICAS

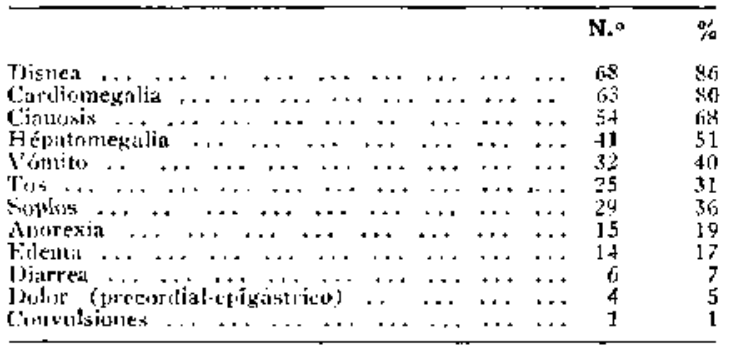

\section{Examen radiológico}

Los hallazgos radiológicos dependerán del grado de compromiso del corazón y de la presencia o ausencia de insuficiencia cardíaca.

El agrandamiento del ventrículo izquierdo se manifiesta en los primeros momentos por elongación y aumento de la convexidad del arco inferior izquierdo, con la punta descendida. En los grados más avanzados hay un franco aumento de la silueta cardíaca hacia la izquierda y hacia atrás, siendo este úl timo visualizado en la posición O.I.A., en la cual se vé la ocupación del espacio retrocardíaco. En los agrandamientos muy acentuados del ventriculo izquierdo hay igualmente un aumento de la sombra cardíaca hacia la derecha, por desplazamiento de las cavidades derechas hacia ese lado.

Cuando la fibroelastosis afecta las valvas de la mitral, produdiendo una estenosis, Ia aurícula izquierda se agranda, lo que se observa especialmente en O.D.A., mediante la papilla baritada.

Si el paciente es visto en insuficiencia cardíaca, la silueta del corazón tiende a asumir un aspecto triangular debido al marcado agrandamiento en todas direcciones, pero el ventrículo izquierdo siempre se hace manifiesto, dando a la silueta cardíaca un aspecto globuloso.

Los cambios radiológicos pulmonares consisten en la congestión vascular de los hilios, con acentuación de las sombras vasculares en los vértices y en las bases. Con la progresión del cuadro el pulmón toma un aspecto irregularmente moteado.

Prec y Gassels ", al igual que Gasul y col. "i, tuvieron oportunidad de practicar angiocardiografías en tres enfermos de fibroeslastosis, observando agrandamiento del ventriculo izquierdo y retardo en el vaciamiento de esta cavidad. 
Adams y Katz ' realizaron cateterismo cardíaco en cuatro pacientes encontrando en dos de ellos cifras discretamente elevadas de la presión en el ventrículo derecho $\mathrm{y}$ arteria pulmonar.

Si bien es cierto que esta enfermedad se presenta en la mayor parte de los casos con iniciación brusca y dramática, es posible, con un interrogatorio exhaustivo y preciso, o por un examen precoz casual, demostrar que los sintomas se inician hastante ante que los accidentes agudos. Así, todo lactante que presente pequeños accesos de cianosis o de disnea mal explicados, o pequeños síncopes inesperados acompañados muchas veces de tos persistente, $o$ de alteraciones gastrointestinales como vómitos con retardo de la curva ponderal, debe ser sometido a un examen radiológico sistematízado, que al demostrar agrandamiento cardíaco nos permiten sospechar y pensar en la posibilidad de una fibroelastosis.

Estos pequeños accidentes se van repitiendo, con carácter cada vez más grave, como cuadros de neumopatías agudas o discretos desfallecimientos cardíacos, a menudo rápidamente recuperables por los antibióticos, el oxígeno y la digital, que restablecen el equílibrio por algún tiempo, hasta llegar el día en que la gravedad de los síntomas, ya irreductibles. ocasionan la muerte en días u horas.

En general, se puede decir que la evolución, a partir del periodo agudo, conduce a una muerte rápida en la mayor parte de Ios casos, siendo todas las medidas terapéuticas clásicas de la insuficiencia cardíaca, ineficaces. Hill y Reilly ${ }^{*}$, basados en que la fibroelastosis del endocardio pertenece al grupo de las enfermedades del colágeno, han propuesto últimamente el tratamiento de ésta con cortisona, terapéutica que ha fracasado en un caso publicado por Lust y col. "y en uno de nuestros pacientes, en que intentamos dicho tratamiento, no así en un enfermo de Schwejsguth y Nouallie ${ }^{10}$, autores que han observado una remisión de la sintomatología hasta el presente, lo que los hace preguntarse si se trata de una remisión espontánea, transitoria, o si en realidad el enfermo ha curado definitivamente; la exactitud del diagnóstico queda insegura en ausencia de la comprobación anatómica, que es la única indiscutible.
Por otra parte, no es fácil este planteamiento ya que la mayor parte de los enfermos llegan al hospital en plena fase terminal, como sucedió en nuestro caso y en el de Lust y col. ", lo que no daría tiempo a la cortisona para actuar.

\section{Diagnóstico diferencial}

Los siguientes cuadros deben considerarse en el diagnóstico diferencial: malformaciones congénitas graves del corazón y grandes vasos, miocarditis intersticial, enfermedad glucogénica del corazón, deficiencias vitamínicas y rabdomioma.

Las malformaciones graves del corazón y de los grandes vasos son sin duda la principal causa de agrandamiento cardiaco en el niño y de éstas sólo debemos considerar las que producen hipertrofia del ventrículo izquierdo. La presencia de latidos femorales eliminan la coarctación de la aorta y la ausencia de soplos en la base el ductus o un defecto del septum aórtico, al igual que la estenosis aórtica o sub. aórtica. Las anomalías más complejas, como la atresia tricuspídea, pueden eliminarse por la cianosis permanente y los hallazgos radiológicos.

El origen anómalo de la coronaria izquierda es quizás el diagnóstico diferencial más difícil que se puede plantear, y es el estudio del electrocardiograma (signos de infarto miocárdico) de valiosa ayuda en la diferenciación de estos dos cuadros si bien algunos autores, como Mc Kinley, Andrews y Neill ${ }^{11}$ y Lambert y col. ${ }^{1 *}$, encuentran lesiones electrocardiográficas similares en ambos.

Respecto a la miocarditis intersticial de Fiedler, hay que señalar que en esta afección el agrandamiento cardíaco es siempre debido a dilatación más que a hipertrofia y nunca existe en forma aislada o predominante de una cavidad. El diagnóstico diferencial con la enfermedad glucogénica cardíaca se puede hacer por la biopsia muscular, que demuestra el deposito de glucógeno.

La carencia de tiamina (corazón BeriBeri) y de ácido ascórbico han sido descritas como causa de agrandamiento cardíaco. En estos casos se trata siempre de niňos con distrofia acentuada y el agrandamiento es debido a dilatación de las cavi. dades, con o sin hipertrofia del ventriculo derecho. En lo que se refiere al rabdomio- 
ma, este tumor es localizado y muy frecuentemente múltiple.

Finalmente, respecto a patogenia, Collier y Rosahn ${ }^{1:}$ así como Farber ${ }^{11}$ y Stadler" creen que Ia fibroelastosis sería una secuela de una endocarditis fetal, debida a infección intrauterina. Contra esto está la falta de reacción inflamatoria endo o miocárdica en la mayor parte de los casos estudiados.

Algunos autores, como Disman ${ }^{15}$, Gross ${ }^{\text {t: }}$, Weimberg e Himelfarb ${ }^{t}$ y Edmons y Selye ${ }^{17}$ son partidarios de una teoría malformativa por alteración en el desarrollo bulbo-ventricular, aduciendo como prueba la frecuente asociación de fibroelastosis con otras malformaciones cardíacas y al hecho de haber sido descrita en dos hermanos. Como ya hemos dicho, para Hill y Reilly ${ }^{8}$ se trataría de una verdadera enfermedad del colágeno, quedando sin explicar el por qué en esta enfermedad el trastorno queda estrictamente localizado al corazón.

Todas estas hipótesis tienen a su favor argumentos serios pero también objeciones no menos importantes, lo que hace difícil, en el estado actual de nuestros conocimientos, decir quien está en la razón, con lo que queda abierto un interrogante que sin duda futuras investigaciones lograrán eselarecer.

\section{RESUMEN}

Se presentan dos casos de fibroelastasis del endocardio y se revisan otros 77 publicados en la hiteratura extranjera, con análisis de su anatomía patológica, clínica y etiologia.

\section{SUMMARY}

Two cases of Endocardial Fibroelastosis, with postmortem examination, are reported. A review of literature is added.

\section{BIBLIOGRAFÍA}

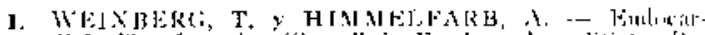

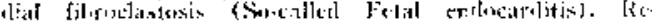

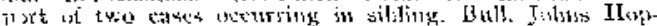
iviss frost. $72: 290,1943$.

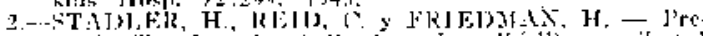

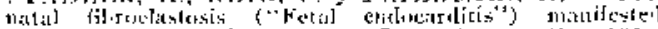

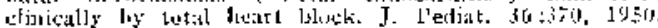

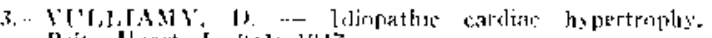
Brif. Hett J. प: $14,19.17$.

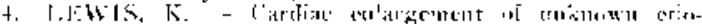

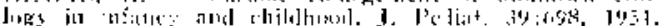

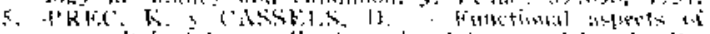

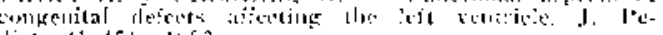
iliat. $41: 451 . \quad 1+20$.

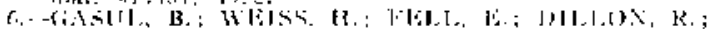

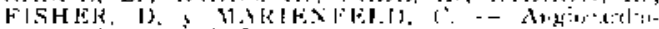

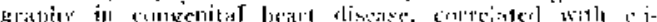

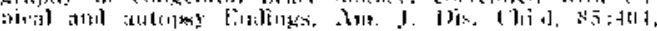
josi

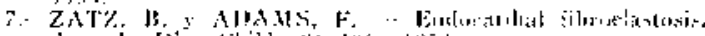

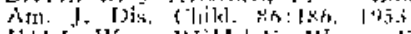

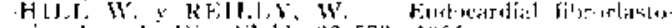

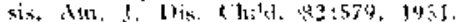

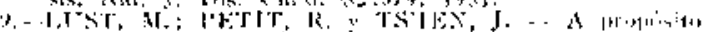

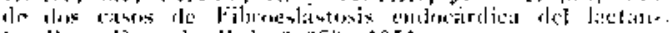

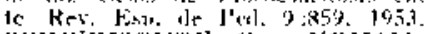

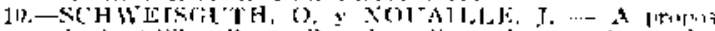

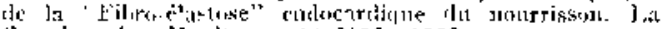

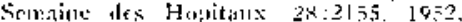

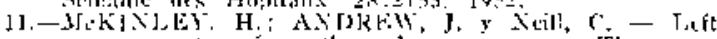

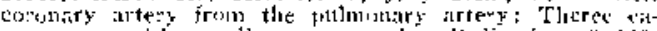

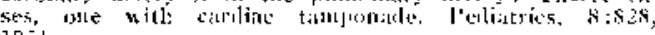
19 कें।

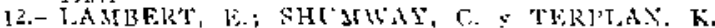

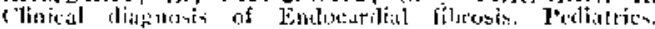
$11: 255.1453$

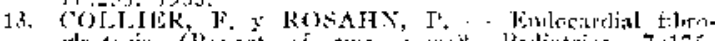
aliatosis (Report of two citstst. l’ctliatries. $7: 175$, 1951 .

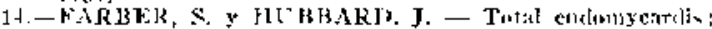

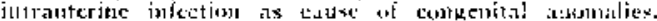

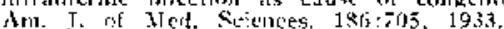

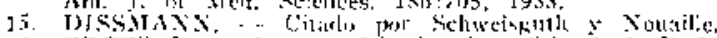

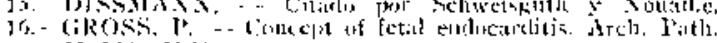

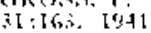

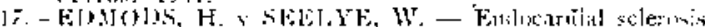

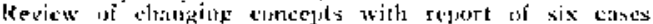

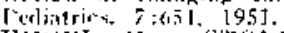

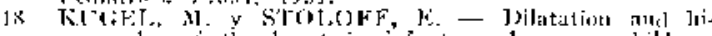

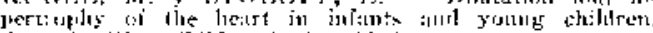
Am. O. Wis. ('thith. +5:829. 19333.

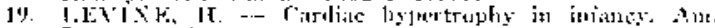

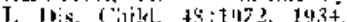

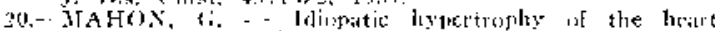

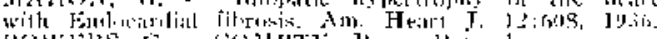

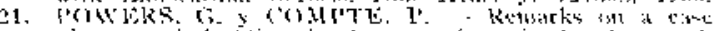

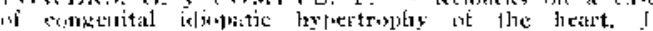

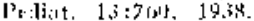

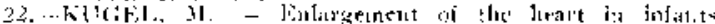

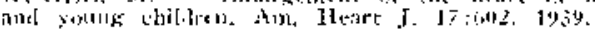

23.

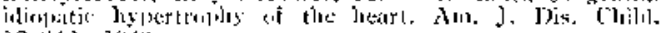
$79: 3+2,30+1)$.

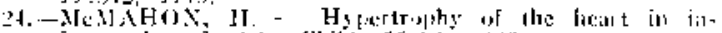

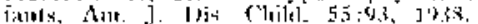

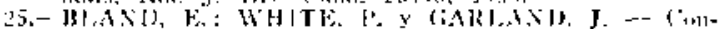

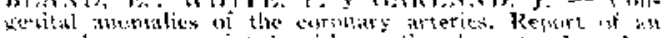

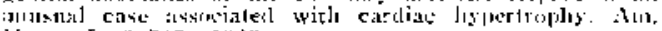

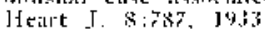

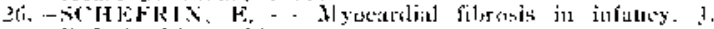

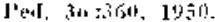

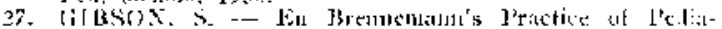
tries. vint. 0

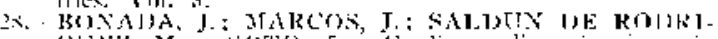
(jLiz, if y

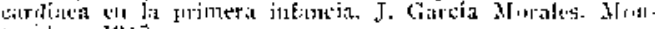
evicla $\mathrm{F}^{1-1} \overrightarrow{\mathrm{s}}$

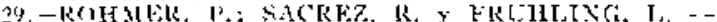

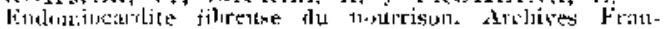

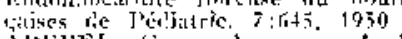

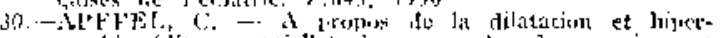

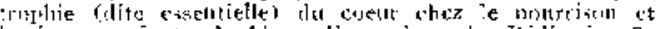

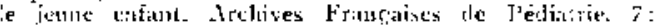
i. 52.19 . 19.

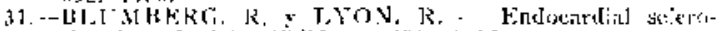

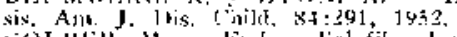

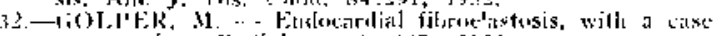

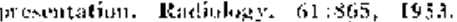

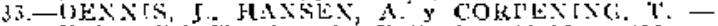

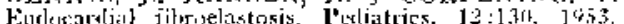

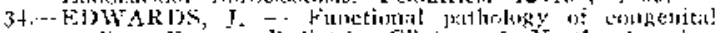
Hatiac lisetse. Pediatrie Clisics of Sorth Aluerica. Sauniters. Ficlunity, 1y5t. 